\title{
PRODUCTION OF PLANETARY NEBULAE
}

\author{
M.P. Savedoff, G.S. Kutter, and H.M. VAN Horn \\ (C.E.K. Mees Observatory, Dept. of Physics and Astronomy, University of Rochester, \\ Rochester, N.Y., U.S.A.)
}

For various reasons, we have been studying evolution in the pre-white dwarf phase at Rochester. Our attention to the relevance of this work to planetary nebulae came as a result of calculations of the evolution of a one solar-mass iron star carried out at Rochester by S. Vila. Here the neutrino processes drive the peak luminosity to log $L / L_{\odot}=4 \cdot 26$, at an effective temperature $\log T_{\text {eff }}=5 \cdot 53$. Although these models are brighter than $100 L_{\odot}$ for 500000 years, they are brighter than $1000 L_{\odot}$ for 4000 years, and exceed $10000 L_{\odot}$ for 900 years. We are therefore near the luminosity of the planetary-nebula nuclei, but considerably hotter, for a period of the order of the planetary lifetimes. Except for the temperature discrepancy, these models are in rough agreement with the observationally determined evolutionary sequence found by O'Dell (1963) and by Harman and Seaton (1964) and Seaton (1966).

The space density of planetary nebulae and white dwarfs and their Galactic kinematics (to the extent that they are known) are consistent with this evolutionary connection, provided the planetary lifetime is approximately 10000 years.

Let us examine two observational properties of planetaries for guidance in discovering modes of planetary production. Clearly, the expansion velocities in the range of $30-100 \mathrm{~km} / \mathrm{sec}$ are well separated from those of the novae and supernovae, which we may call explosive objects. This flow is characteristic of sound speeds of the order of $10^{5} \mathrm{~K}$ rather than $10^{9} \mathrm{~K}$. We are led to consider models in which things proceed slowly compared to pulsational periods. This does not preclude Rose's results as being applicable. On the time-scale of $10^{3}$ years, a mass of $0.5 M_{\odot}$ can be accelerated to $30 \mathrm{~km} / \mathrm{sec}$ by a mean luminosity of $100 L_{\odot}$ assuming total efficiency. Removing the material from a deep potential well and possible inefficiencies can easily require the available energy to be nearly a thousand-fold higher.

Secondly, there exist some indications that an object may have only one planetary nebula phase in contrast to the behavior of novae. However, if more than one phase does occur, we suggest a minimum recurrence time for episodes of 10000 years. This may be compared with the pulses found by Rose (1966) and Weigert (1966) of $10^{5}$ and 4000 years.

We propose to put nuclear fuel into Vila's stars and watch what happens. These differ from earlier models in including neutrino effects; e.g. that the central temperatures remain below a peak temperature which propagates outwards, increasing in

Osterbrock and O'Dell leds.), Planetary Nebulae, 400406. I. I.U. 
amplitude until it reaches a maximum peak temperature $\log T=8.92$ at $M / M_{*}=0.73$, this peak decreases now in peak temperature as it propagates out to a fractional mass at least as large as 0.99 . The time-scale of non-thermal equilibrium is related to the photon diffusion time,

$$
t \simeq k M / R c \simeq 10^{4} \text { years } .
$$

Models of pure carbon are reported as the simplest system for which the temperature inversion is expected and for which a large temperature-sensitive energy source is available. This last condition causes us to omit models with helium and hydrogen as the energy sources responsible for the flash. The abundance of elements in white dwarfs is uncertain, although one of us has suggested evidence for a mean charge of 6-8 for one group (Van Horn, 1968).

We estimate that for masses less than $1.08 M_{\odot}$, neutrino losses will dominate and hence that no mass loss will occur. In contrast, for $M$ greater than 1.6 $M_{\odot}$, nuclear reactions begin before the temperature peak is formed, hence the energy-generation peak is in the centre. These models should resemble main-sequence models. Calculations have been carried out for $1 \cdot 1 M_{\odot}$ from initial conditions shown in Table 1 ,

\section{Table 1}

\section{Initial conditions for $1 \cdot 1 M_{\odot} \mathrm{C}^{12}$ Star}

$$
\begin{aligned}
& \text { Polytrope of index } 3 \\
& R_{*}=0.4 R_{\odot} \\
& \rho_{\mathrm{c}}=10^{3} \mathrm{~g} / \mathrm{cm}^{3} \\
& T_{\mathrm{c}}=80 \times 10^{\circ} \mathrm{K} \\
& L=10^{3} L_{\odot} \\
& T_{\text {eff }}=46000^{\circ} \mathrm{K}
\end{aligned}
$$

through the white dwarf stage, assuming hydrostatic equilibrium throughout. The initial model is derived distantly from a polytrope and has no degeneracy, no neutrino emission, etc.

Evolution in the $L, T_{\mathrm{e}}$ plane (see Figure 1) is similar to the $M=1 M_{\odot}$ iron model of Vila (1965) except for the second loop driven by the $\mathrm{C}^{12}$ burning. We find that all the nuclear energy is essentially converted into gravitational energy through the work done in expanding the star adiabatically. No real instability (in the sense of Mestel's thermal run-away) occurs.

In Figure 2, note the development of an extended convective shell driven by the extreme peak in the energy generation. The peak rate of surface expansion was only $30 \mathrm{~cm} / \mathrm{sec}$, vanishingly small compared to escape velocities exceeding $10^{8} \mathrm{~cm} / \mathrm{sec}$. Temperature distributions for some of the models listed in Table 2 are shown in Figs. $3 a$ and $3 b$.

Can these models be consistent with mass loss? We had originally expected an extended model, $R=10^{12} \mathrm{~cm}$ with convection throttled in the surface layers, but the 

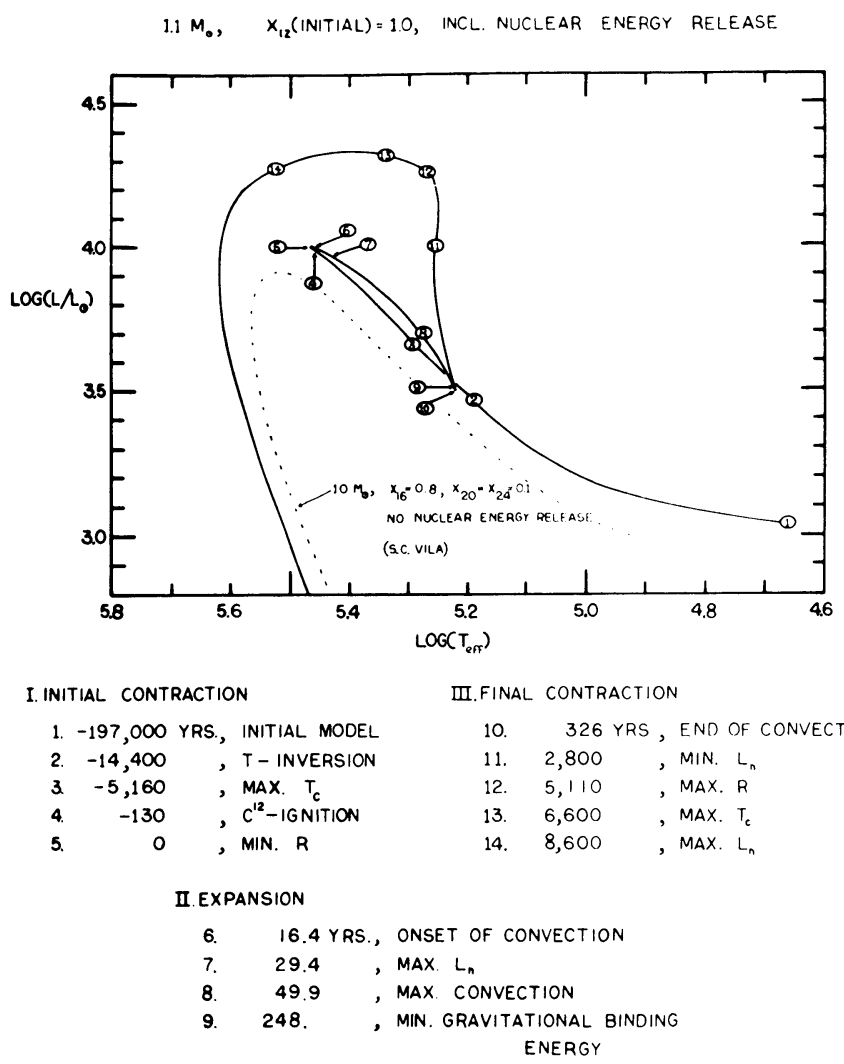

III.FINAL CONTRACTION

FIG. 1. $L, T_{\mathrm{e}}$ diagram for $1.10 \mathrm{M}_{\odot} C^{12}$ star .

model allows convection only out to $M / M_{*}=0 \cdot 96$. Ledoux has suggested that these models should be vibrationally unstable, with pulsation amplitude increasing until damped by mass loss from the surface. We have been unable to follow this idea into the non-linear regions. Rough estimates show that the amplitude must be comparable to the stellar radius for the velocity to reach the escape velocity.

We had hoped for mass loss through a 'stellar wind' analogous to the Parker 'Solar Corona' Model. This 'corona' could be produced by sonic waves generated in the convective region. Lighthill (1952) and Proudman (1952) has shown that the rate of sonic noise generation by an isotropic turbulent medium is approximately

$$
\begin{aligned}
& L_{\mathrm{sonic}} \\
& 4 \pi R^{2}
\end{aligned}=30 \rho v_{\mathrm{s}}^{3}\left(v / v_{\mathrm{s}}\right)^{8} .
$$

Using conventional mixing length theory we estimate $v=2 \mathrm{~km} / \mathrm{sec}$ and $v_{\mathrm{s}}=10^{3} \mathrm{~km} /$ sec, hence $L=10^{26 \cdot 7} \mathrm{ergs} / \mathrm{sec}$. We fall short of the needed energy by a factor of $10^{10}$. 
Seaton (1966) has noted that radiation pressure may dominate the stellar models in the region of the planetary nebula nuclei. Setting

we find for electron scattering

$$
\frac{G M \rho}{r^{2}}=\frac{L \kappa \rho}{4 \pi r^{2} c}
$$

$$
L_{\odot} \sim \frac{10^{4 \cdot 8} M / M_{\odot}}{(1+X)} .
$$

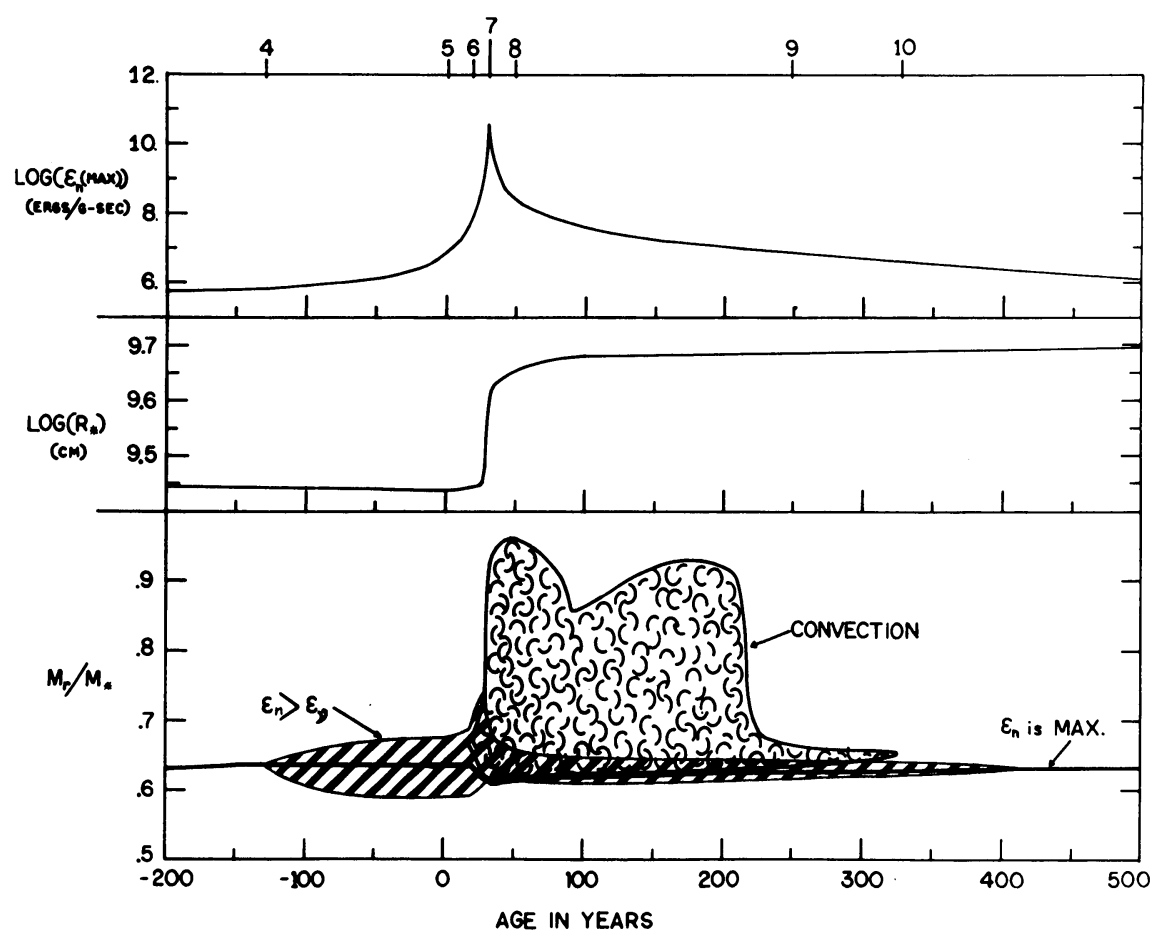
4. $C^{12}-$ IGNITION
8. MAXIMUM CONVECTION
5. MINIMUM $R$
9. MINIMUM GRAVITATIONAL BINDING ENERGY
6. ONSET OF CONVECTION
10. END OF CONVECTION
7. MAXIMUM $\varepsilon_{n}$

FIG. 2. Development of $\log \varepsilon_{\mathrm{n}}(\max ), \log R^{*}$, and extent by mass fraction of nuclear burning $\left(\varepsilon_{\mathrm{n}}>\varepsilon_{v}\right)$ and convection vs. time during the $C^{12}$ flash of the $1 \cdot 1 M_{\odot}$ star.

At the second pass (see Figure 1) peak log luminosity reached 4.33 at that phase where the temperature peak was at $M / M_{*}=0 \cdot 84$.

Thus an increase of the opacity or the luminosity by a factor of 3 would suffice to free the material gravitationally. We have come close. For $L>L_{\mathfrak{c}}$, as a first approxima- 


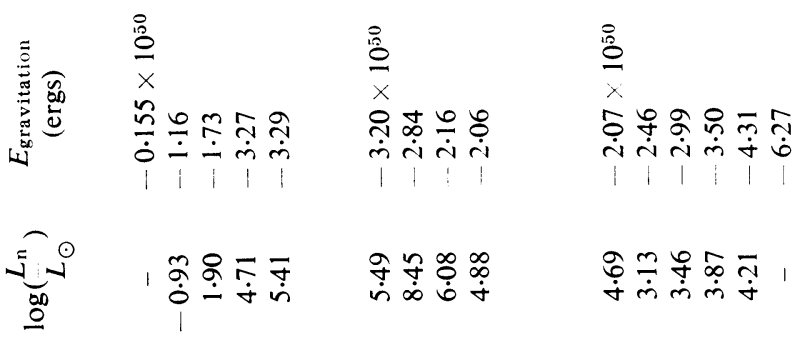

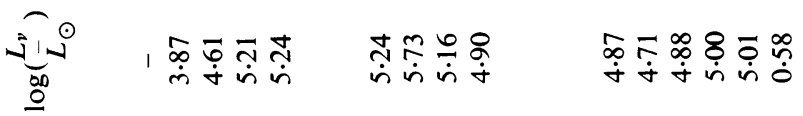

क्ञ

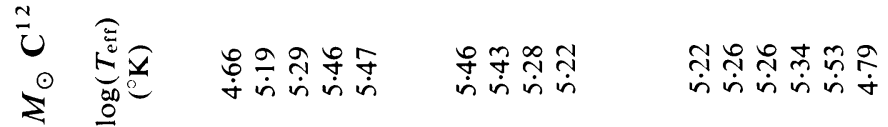

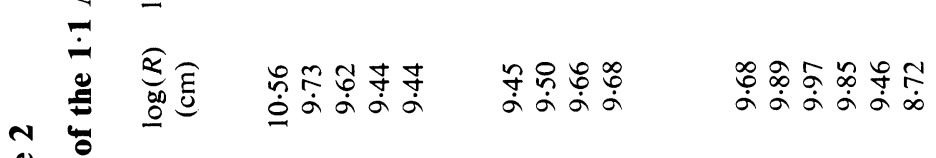

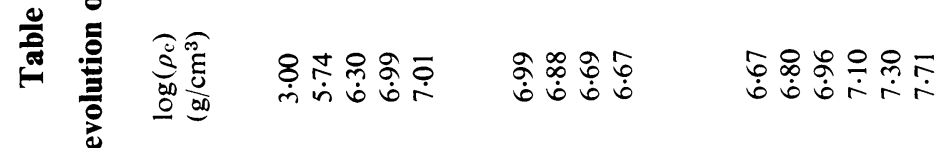

Е

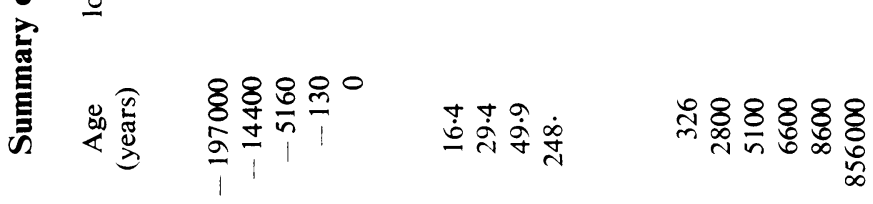

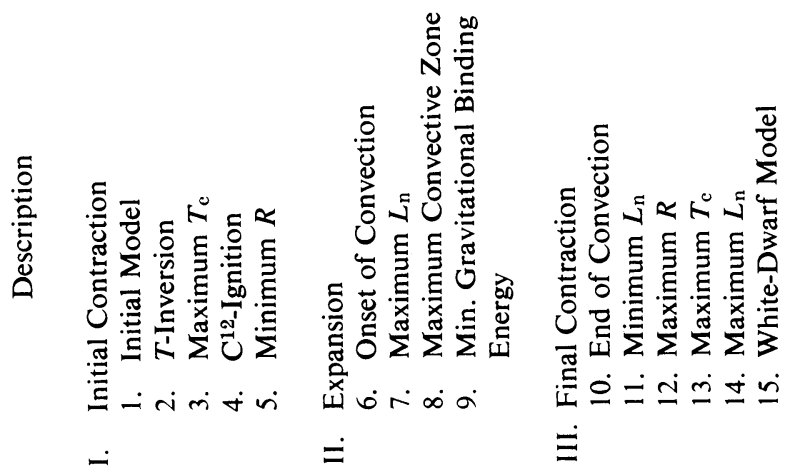




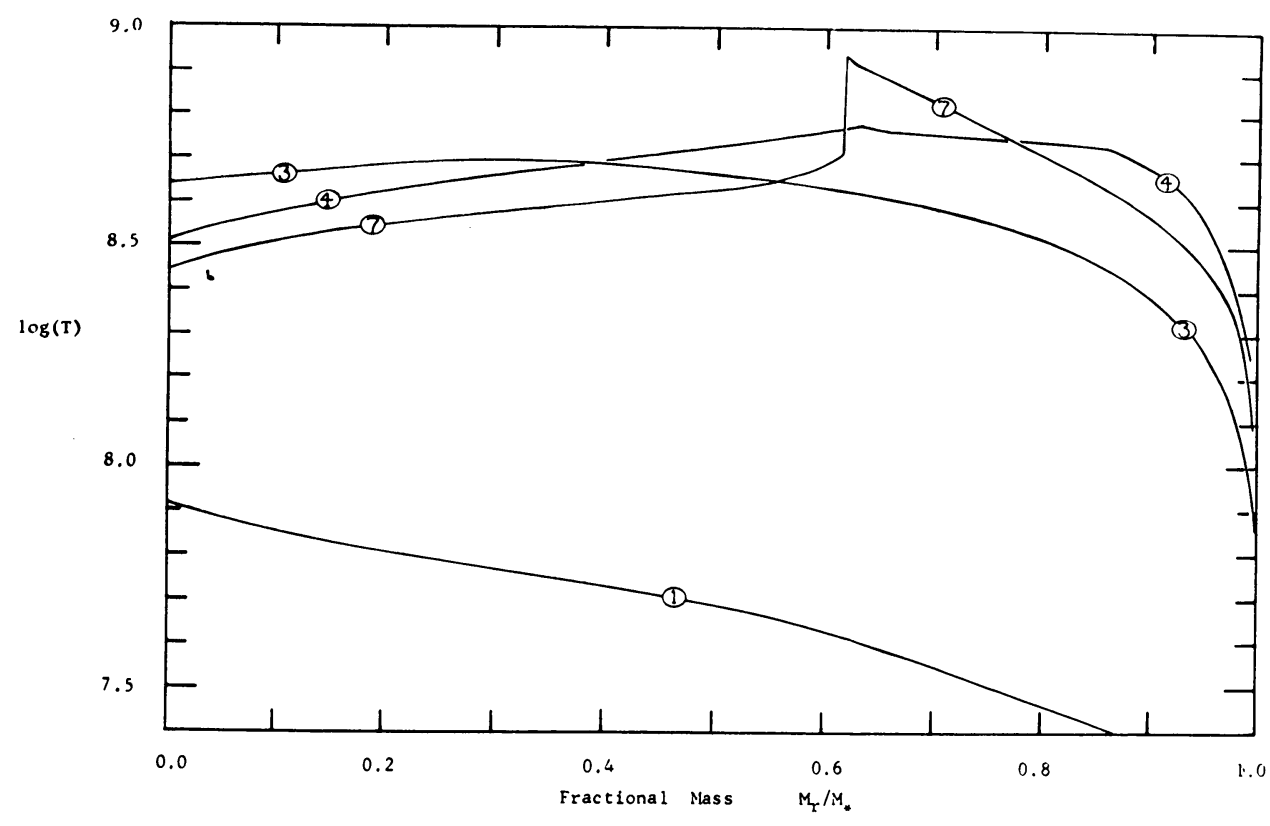
1. -197.000 . yrs.. Initial Model
4. -130. yrs.. $c^{12}$ - Ignition
3. -5.160 . yrs.. Maximum $T_{c}$
7. 29.4 yrs.. Maximum $L_{n}$

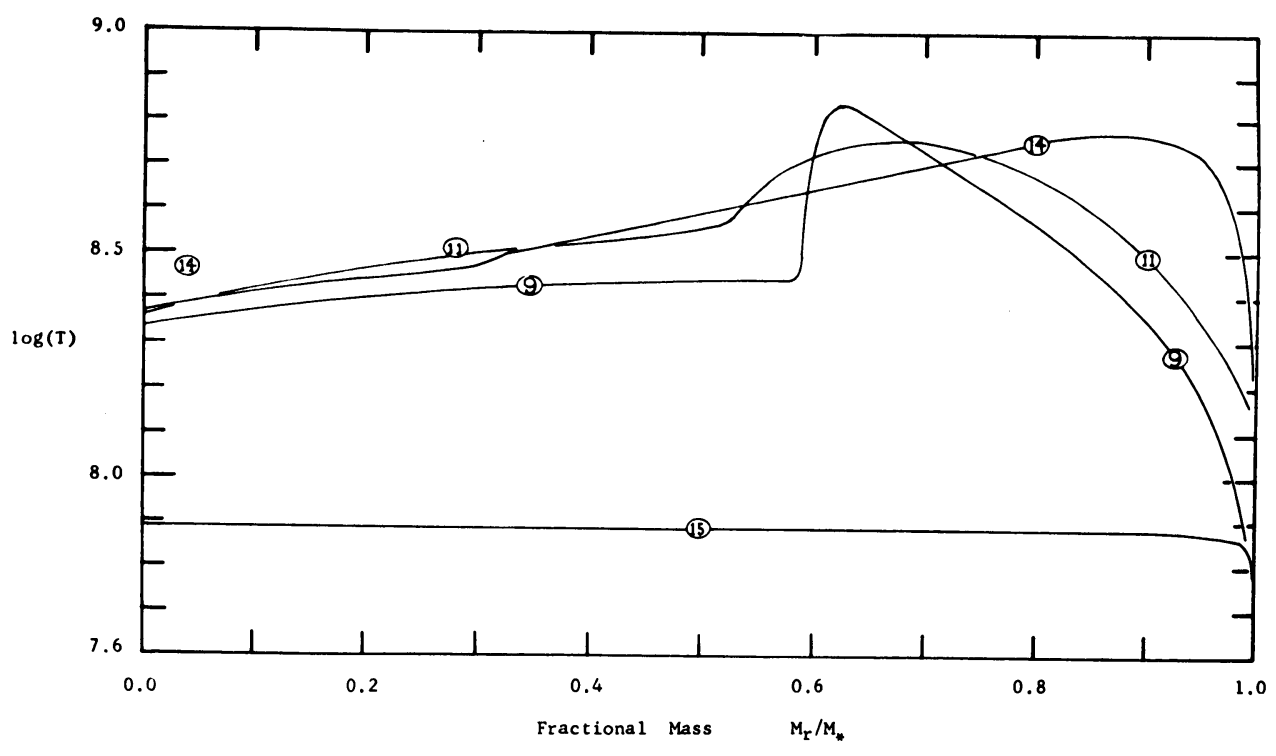
9. 248, yrs., Minimum Gravitational Binding Energy
14. 8,600. yrs., Maximum $\mathrm{L}_{n}$
11. 2,800. yrs., Minimum $\mathrm{L}_{n}$
15. 856,000 . yrs., White Dwarf Model

FIG. 3. Distribution of $\log T$ vs. fractional mass for selected models of the $1 \cdot 1 M_{\odot} C^{12}$ star. 
tion, the behavior of the gas would resemble free flow into a vacuum until such time as its opacity decreased (recombination) or the luminosity decreased so that the gravitational force becomes dominant again.

The apparent discrepancy in effective temperature and hence radius between our models of the planetary nuclei may be removed by judicious additions of low-molecular weight material to the outer layers of the star. To estimate the effects of this inhomogeneity upon the calculations we require temperature continuity at radius $r_{1}=r_{2}$, that

$$
T=\underset{4 \cdot 25}{\beta} \underset{k r_{1}}{H} \mu_{1}\left(1-\frac{r_{1}}{R_{1}}\right)=\underset{4 \cdot 25}{\beta} \frac{H G M}{k r_{1}} \mu_{2}\left(1-\begin{array}{c}
r_{1} \\
R_{2}
\end{array}\right)
$$

be the same both for the homogeneous composition (1) and for the boundary of the outer layer (composition (2)),

$$
\stackrel{R_{2}}{R_{1}}=\frac{1}{\mu_{1}}-\left(\begin{array}{l}
\mu_{1}-1 \\
\mu_{2}
\end{array}\right) \begin{aligned}
& R_{1} \\
& r_{1}
\end{aligned} .
$$

In Table 3 we see that these radii can be appreciably enlarged by the substitution of a $1 \%$ helium envelope or a $10^{-5} \%$ hydrogen envelope.

\begin{tabular}{|c|c|c|c|c|c|}
\hline $\log T$ & $R_{1} / r_{1}$ & $R_{2} / R_{1}$ & $\log M / M \star$ & $\log \rho$ & $\kappa$ \\
\hline \multicolumn{6}{|c|}{ Helium-Carbon } \\
\hline $8 \cdot 28$ & $3 \cdot 6$ & 3.9 & .004 & $3 \cdot 11$ & 0.14 \\
\hline $8 \cdot 14$ & $3 \cdot 0$ & $2 \cdot 3$ & -.002 & $2 \cdot 62$ & $\cdot 16$ \\
\hline \multicolumn{6}{|c|}{ Hydrogen-Carbon } \\
\hline 6.93 & 1.42 & $x$ & $-2 \times 10^{7}$ & -1.37 & .26 \\
\hline $6 \cdot 78$ & $1 \cdot 38$ & 12. & $-5 \times 10^{-8}$ & -1.92 & $0 \cdot 30$ \\
\hline
\end{tabular}

\section{Table 3}

In summary, we have made another pass through the region of the planetary nuclei; we have come close, but we did not find a nebula. Proposed extensions of this work are being investigated as they seem likely to provide possible planetaries.

\section{References}

Harman, R.J., Seaton, M.J. (1964) Astrophys. J., 140, 824.

Lighthill, M.J. (1952) Proc. R. Soc. London, A211, 564.

Proudman (1952) Proc. R. Soc. London, A214, 119.

O'Dell, C.R. (1963) Astrophys. J., 138, 67.

Rose, W. K. (1966) Astrophys. J., 146, 838.

Seaton, M.J. (1966) Mon. Not. R. astr. Soc., 132, 113.

Van Horn, H. M. (1968) Astrophys. J., 151, 227.

Vila, S. (1965) Thesis, University of Rochester.

Weigert, A. (1966) Z. Astrophys., 64, 395. 